\title{
OPEN NB-LRR-encoding genes conferring susceptibility to organophosphate pesticides in sorghum
}

\author{
Zihuan Jing ${ }^{1}$, Fiona Wacera W. ${ }^{1}$, Tsuneaki Takami $^{1}$, Hideki Takanashi ${ }^{2}$, Fumi Fukada ${ }^{1}$, \\ Yoji Kawano ${ }^{1}$, Hiromi Kajiya-Kanegae ${ }^{3}$, Hiroyoshi Iwata ${ }^{2}$, Nobuhiro Tsutsumi ${ }^{2}$ \& \\ Wataru Sakamoto ${ }^{1 凶}$
}

Organophosphate is the commonly used pesticide to control pest outbreak, such as those by aphids in many crops. Despite its wide use, however, necrotic lesion and/or cell death following the application of organophosphate pesticides has been reported to occur in several species. To understand this phenomenon, called organophosphate pesticide sensitivity (OPS) in sorghum, we conducted QTL analysis in a recombinant inbred line derived from the Japanese cultivar NOG, which exhibits OPS. Mapping OPS in this population identified a prominent OTL on chromosome 5, which corresponded to Organophosphate-Sensitive Reaction (OSR) reported previously in other mapping populations. The $O S R$ locus included a cluster of three genes potentially encoding nucleotide-binding leucine-rich repeat (NB-LRR, NLR) proteins, among which NLR-C was considered to be responsible for OPS in a dominant fashion. NLR-C was functional in NOG, whereas the other resistant parent, BTx623, had a null mutation caused by the deletion of promoter sequences. Our finding of $O S R$ as a dominant trait is important not only in understanding the diversified role of NB-LRR proteins in cereals but also in securing sorghum breeding free from OPS.

Crops growing under natural conditions face the threats from different environmental factors, among which pathogens and pests often cause a serious loss of yield ${ }^{1-3}$. To counteract such biotic stresses, breeding crops that confer resistance to them is demanding ${ }^{4,5}$. Plants are known to defense against pathogens by immune responses with $R$ genes $^{6-8}$. The majority of genetically characterized disease resistance traits map to $R$ genes encoding nucleotide-binding domain (NB) and leucine-rich repeat (LRR) proteins, and they act as receptors to perceive effectors derived from pathogens and to activate effector-trigger immunity (ETI), which includes cell death and reactive oxygen species production ${ }^{9-16}$. In addition to harnessing these resistant genes, chemical management of pests is a practical way; plants are applied by the pesticides to manage the insects that attack a broad range of crop species ${ }^{17,18}$. Controlling aphids, thrips and other pests are important not only to avoid yield loss by severe infestation but also to prevent these insects from spreading vector-borne diseases ${ }^{19-22}$.

Organophosphates are a group of commonly used pesticides that kill insects by causing neurotoxicity through the inhibition acetylcholinesterase activity, whereas these compounds show no toxicity to host plants ${ }^{23}$. Cell death or leaf injury that resembles the hypersensitive reaction to pathogens, however, is rarely observed when crops are sprayed with a particular type of organophosphate pesticides ${ }^{24}$. Molecular analysis of such organophosphate pesticide sensitivity (OPS) was first reported in tomato; plants harboring the Pto locus (conferring resistance to Pseudomonas syringae pv. tomato strains) exhibited necrosis after being sprayed with fenthion, an organophosphate pesticide ${ }^{25-27}$. This OPS phenotype in tomato was shown to depend on the Fen gene, encoding a serinethreonine protein kinase belonging to the receptor-like kinases among the $R$ genes $^{28}$. More recently, another nucleotide-binding leucine-rich repeat (NB-LRR) gene termed Prf embedded within the Pto/Fen gene cluster was shown to act in concert with Pto/Fen to activate multiple plant-pathogen signal transduction pathways ${ }^{29-31}$.

The other example of NB-LRR responding to organic chemicals is VICTR in Arabidopsis, which responds to accession-specific root growth arrest with the small-molecule [5-(3,4-dichlorophenyl) furan-2-yl]-piperidine-1-ylmethanethione (DFPM) ${ }^{32,33}$. These findings present the intriguing recognition mechanism of external chemicals through NB-LRR, whereas the cases are limited to several species. In this study, we focused on another example of OPS in sorghum, which has segregated in a recombinant inbred population we recently established.

\footnotetext{
${ }^{1}$ Institute of Plant Science and Resources, Okayama University, 2-20-1 Chuo, Kurashiki, Okayama 710-0046, Japan. ${ }^{2}$ Graduate School of Agricultural and Life Sciences, The University of Tokyo, 1-1-1 Yayoi, Bunkyo-ku, Tokyo 113-8657, Japan. ${ }^{3}$ Research Center for Agricultural Information Technology, National Agriculture and Food Research Organization, Minato-ku, Tokyo 105-0003, Japan. ${ }^{\circledR}$ email: saka@okayama-u.ac.jp
} 
a

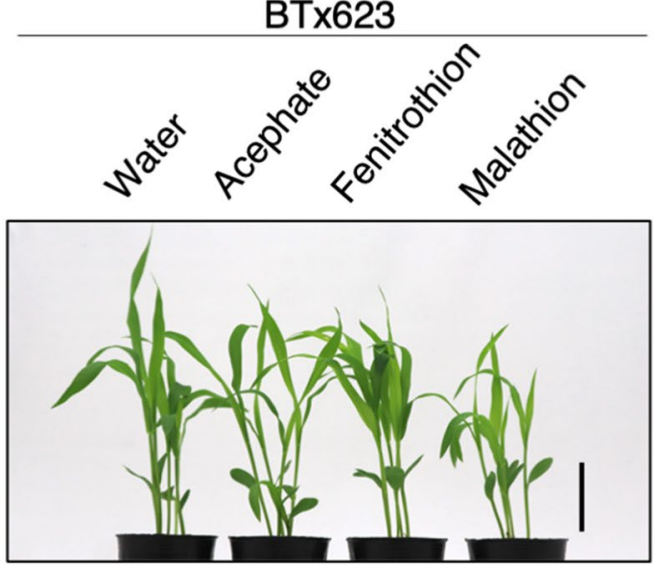

b

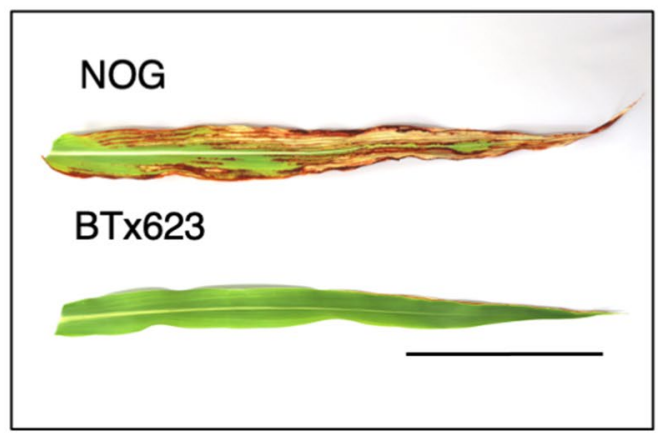

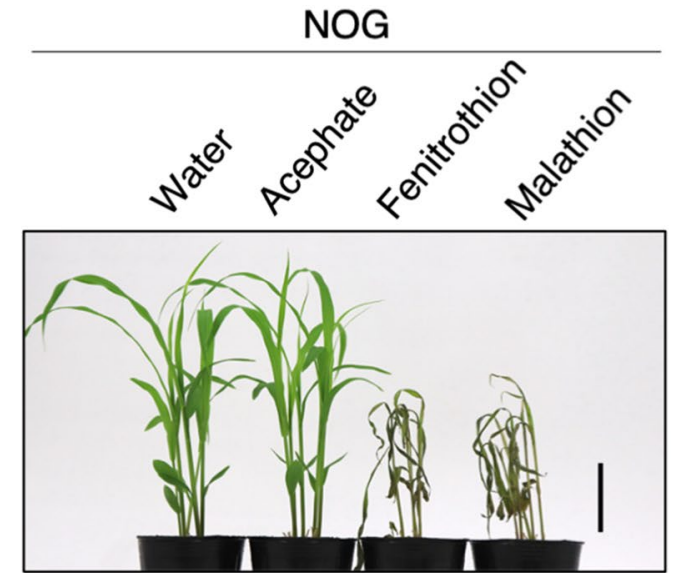

c

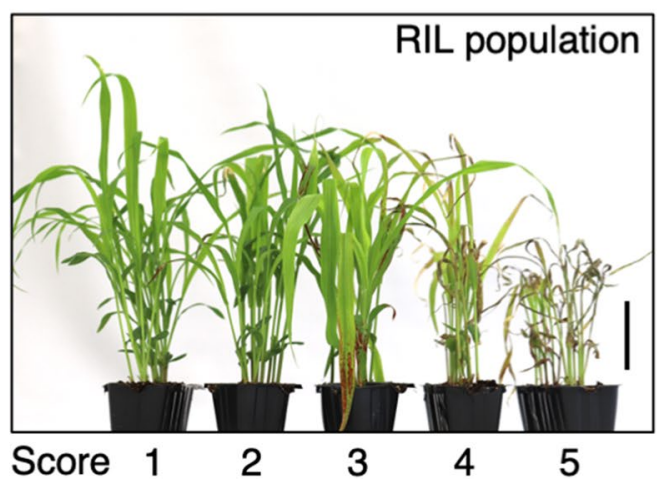

Figure 1. Phenotype of sorghum plants with organophosphate pesticide treatment. (a) Symptoms of BTx623 (left) and NOG (right) after acephate, fenitrothion, and malathion treatment. Scale bars; $5 \mathrm{~cm}$. (b) OPS phenotypes of NOG and BTx623 in flag leaves, after spraying fenitrothion (1:500). Scale bars; $10 \mathrm{~cm}$. (c) The phenotyping pool of OPS phenotypes segregating in the RIL population at the seedling stage. The mixture lines in each score with fenitrothion (1:500) spraying are displayed. Scale bars; $5 \mathrm{~cm}$.

Sorghum (Sorghum bicolor L. Moench), is the fifth most important cereal crop worldwide ${ }^{34}$, and its high biomass along with extreme tolerance to drought and high-temperature conditions makes sorghum a potential crop for food, fodder, and bioenergy production ${ }^{35}$. Like other crops, sorghum is often devastated by aphid infestations and the organophosphate pesticides are routinely used. During the sorghum growing period from May to August that includes rainy season in Japan, an outbreak of the major aphid Melanaphis sacchari (Zehntner) occurs in our test field and elsewhere in Japan ${ }^{36,37}$. Its reproductive capacity is extremely high, and the plants result in turning leaves into rusty brown and necrosis, eventually leading to plant death ${ }^{38}$. In order to control the aphid population effectively, we use organophosphate pesticides, some of which were then shown to cause OPS and result in brown spotting lesion both in leaves and stems. This OPS appeared to derive from one Japanese cultivar NOG, whose generic name is Takakibi (alternatively called 'Morokoshi' or 'Koryan', the latter is the Chinese sorghum named 'Gaoliang' in Japanese).

To investigate OPS in sorghum further, we utilized a recombinant inbred line (RIL) population, generated by a cross between BTx623 and NOG ${ }^{39,40}$. Our previous studies indicated that NOG and BTx623 differ not only in morphological traits such as days to heading and plant height but also in photosynthetic response to high temperature and strong light around the heading period ${ }^{39,41}$. The development of genetic map with high-density markers (>3700), generated by RAD-seq in this population allowed us to identify genes responsible for segregating traits as QTLs. Therefore, we considered that this RIL population was suitable for a comprehensive study of OPS in sorghum. Our genetic analysis identified a QTL on chromosome 5 that conferred leaf OPS, which corresponded to the Organophosphate-Sensitive Reaction (OSR).

\section{Results}

Assessing organophosphate pesticide sensitivity. BTx623 and NOG displayed different responses to several types of organophosphate pesticides. Acephate (Ortran) was commonly used in our greenhouse to control aphids, and we noticed no visible growth defect throughout the entire growth period of both NOG and BTx623. In contrast, fenitrothion (Sumithion) and malathion (Marathion) caused severe necrosis only in NOG when applied to seedlings (Fig. 1a, chemicals structures of each pesticide are shown in Supplementary Fig. S1). This sensitivity was apparently holistic when fenitrothion was applied to field-growing NOG (Supplementary Fig. S2), excluding the possibility that OPS is confined developmentally. Necrotic lesions were also apparent 
when pesticides were spotted onto leaves at later growth stages (Fig. 1b). Indeed, we observed this OPS segregating in the RIL population, which prompted us to perform QTL analysis (Fig. 1c).

QTL analysis of OPS using $\mathrm{F}_{9}, \mathrm{~F}_{10}$, and $\mathrm{F}_{11}$ populations from the RIL showed a single peak near marker Chr05:8,666,663 on chromosome 5 after fenitrothion treatment (Fig. 2). An identical peak near marker chr05:8,666,663 was obtained on chromosome 5 with malathion treatment (Supplementary Fig. S3). The location of this QTL, termed $q O P S 5$, on chromosome 5 was similar to those obtained in previous studies, including organophosphate resistance $(o p r)^{42,43}$, resistance to chemical burning $(r c b)^{44,45}$, crop injury ${ }^{24}$, and organophosphatesensitive reaction $(o s r)^{46}$, although the inbred lines used in each study differed. Given that no other prominent QTLs other than those on chromosome 5 were detected, we concluded that OPS in the RIL population is predominantly controlled by this single locus, qOPS5.

Fine mapping. The BTx623xNOG RIL population consists of 213 individuals, in which the genotyping of 3710 markers was determined in the $\mathrm{F}_{6}$ generation $^{39}$. Displaying graphical genotypes of each RIL along with its OPS phenotype allowed us to narrow down the genomic region conferring OPS to a 743-kb region (Fig. 3a) between two SNP markers, Chr05:7,971,429 and Chr05:8,714,734. QTL analysis also showed that the QTL peaks were located at SNP marker Chr05: 8,666,663, encompassed by the chromosome regions detected from the recombination points (Fig. $3 \mathrm{~b}$ ). Within this region, there are 36 genes annotated in the database (https:// phytozome.jgi.doe.gov, Phytozome version 12), in which a cluster of three NB-LRR genes (Sobic.005G071700, Sobic.005G071900, and Sobic.005G072000) existed in a tandem orientation (Fig. 3b, Supplementary Table S1). In fact, this cluster of three NB-LRR genes was reported as being likely to be responsible for leaf injury by Boyles et al. (2017) and for OPS of inbred line Nakei MS3B ${ }^{46}$. In this study, we termed it OSR in accordance with Kawahigashi et al. (2020) and further characterized these NB-LRR genes.

Characterization of the OSR locus in NOG. We investigated the OSR locus in NOG, based on resequencing data of NOG we determined previously (DDBJ Sequence Read Archive Accession No. DRA008159). For simplicity, we termed three NB-LRR genes Sobic.005G071700, Sobic.005G071900, and Sobic.005G072000 as $N L R-A, N L R-B$, and $N L R-C$, respectively. First, comparison of these three genes with those in BTx623 showed that NLR-A was missing in NOG, lacking the corresponding genomic region from 8,457,439 to 8,557,269 on chromosome 5 , which amounted to $\sim 99.8 \mathrm{~kb}$ (Fig. 3b). Lack of $N L R-A$ was subsequently confirmed by PCR specifically amplifying $N L R-A$ (Fig. 3c). Given that OPS is dominant, involvement of $N L R$ - $A$ was unlikely (see below). Second, comparison of NLR-B and NLR-C indicated that both genes existed in NOG and BTx623. The Sorghum bicolor v3.1.1 database (https://phytozome.jgi.doe.gov, Phytozome version 12) predicted that NLR-B had no intron and encoded a 933 amino acid-long protein that had a nucleotide binding site domain (176-457 aa) and a leucine-rich repeat domain (483-903 aa). Similarly, NLR-C contained one exon of 2739 bp encoding 912 amino acids in both NOG and BTx623. We found that in NOG, NLR-B and NLR-C had two and ten amino-acid substitutions, respectively (Fig. 3d). For NLR-B, two amino-acid substitutions, I289V and S650R, were within the NB domain and LRR domain, respectively. The other polymorphism we detected in NOG was a 593-bp insertion, in the 5' upstream region of NLR-C (Fig. 3b). Phylogenetic analysis of NLR-B and NLR-C proteins along with other NB-LRR proteins showed that NLR-B and NLR-C are structurally related to RPM1, which recognizes the AvrRpm1 type III effector avirulence protein from P. syringae in Arabidopsis ${ }^{47}$. They were also closely related to NB-LRRs in Oryza sativa, which leads to lesions on the leaf blade and broad-range resistance to the fungal pathogen Pyricularia oryzae (syn. Magnaporthe oryzae) and the bacterial pathogen Xanthomonas oryzae pv. oryzae, together with strong growth reduction (OsRLR1) $)^{48}$, and rice blast resistance (Pid3) ${ }^{49}$. (Supplementary Fig. S4).

Segregation analysis. We next tested whether OSR in NOG confers susceptibility in a dominant fashion as reported previously. Followed by confirmation of the $\mathrm{F}_{1}$ genotype by PCR, we investigated OPS in each $\mathrm{F}_{1}$ plant (Fig. 4). The results showed that the response to fenitrothion at the seedling stage was somewhat less severe and variable, whereas lesions were clearly observed on the mature leaves in all $\mathrm{F}_{1}$ plants. Although the precise reason for the unstable appearance of lesions at the seedling stage was unclear, it was apparent that all $\mathrm{F}_{1}$ plants exhibited OPS; based on these observations, we concluded that OPS is semi-dominant.

To examine if the observed polymorphisms mentioned previously were indeed linked to the OPS phenotype, segregation analysis was performed. Our survey of RIL individuals showing intermediate OPS scorings in the $\mathrm{F}_{8}$ population found that RIL25 showed segregation of the OSR locus. Therefore, $\mathrm{F}_{9}$ progeny derived from an $\mathrm{F}_{8}$ plant heterozygous for the NB-LRR cluster was subjected to further segregation analysis. A total of 86 plants of RIL25 from the $\mathrm{F}_{9}$ generation were treated with fenitrothion at the heading stage in a greenhouse. Phenotyping of these plants indeed showed the segregation (Fig. 5a). Linkage analysis with genotyping data with $N L R-A$ and NLR-C indicated that OSR segregated in a Mendelian fashion (Fig. 5b,c). Most importantly, co-segregation between OPS and NOG genotypes was found (Fig. 5d). These results strongly supported the notion that the OSR locus is semi-dominant and linked with $N L R-B$ and $N L R-C$.

Transcript accumulation of NB-LRR genes indicates altered expression in NLR-C. We performed semi-quantitative RT-PCR using gene-specific primers in the segregating RIL25 population along with the parental lines (Fig. 6a). RNA was extracted from mature leaves, either before or after fenitrothion treatment, and subjected to RT-PCR. For $N L R-A$, its transcript levels appeared to be extremely low and were not detectable in any samples irrespective of the presence (BTx623) or absence (NOG) of the gene. For NLR-B, we observed 
a

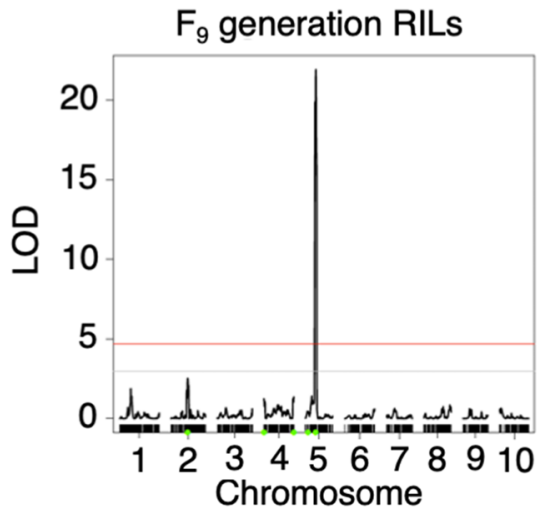

$\mathrm{F}_{10}$ generation RILS

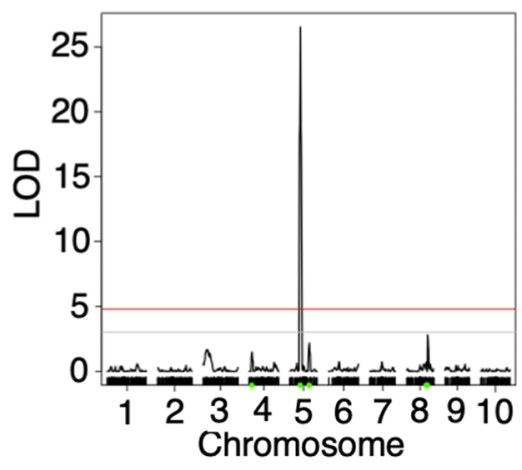

$F_{11}$ generation RILs

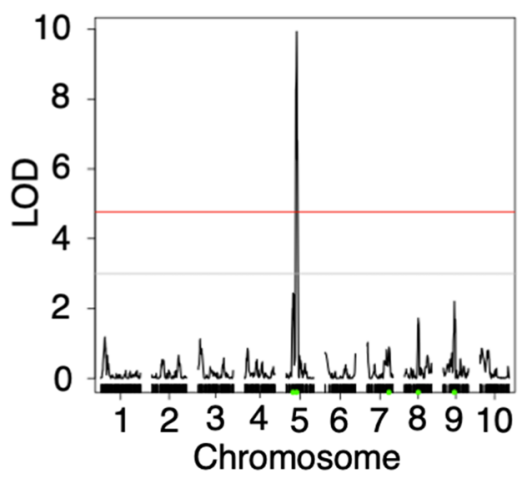

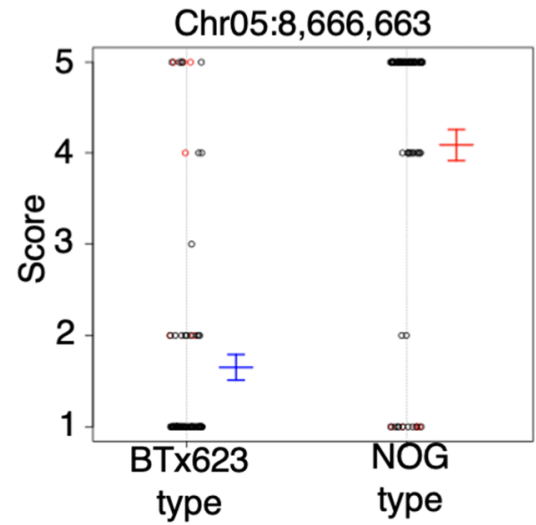
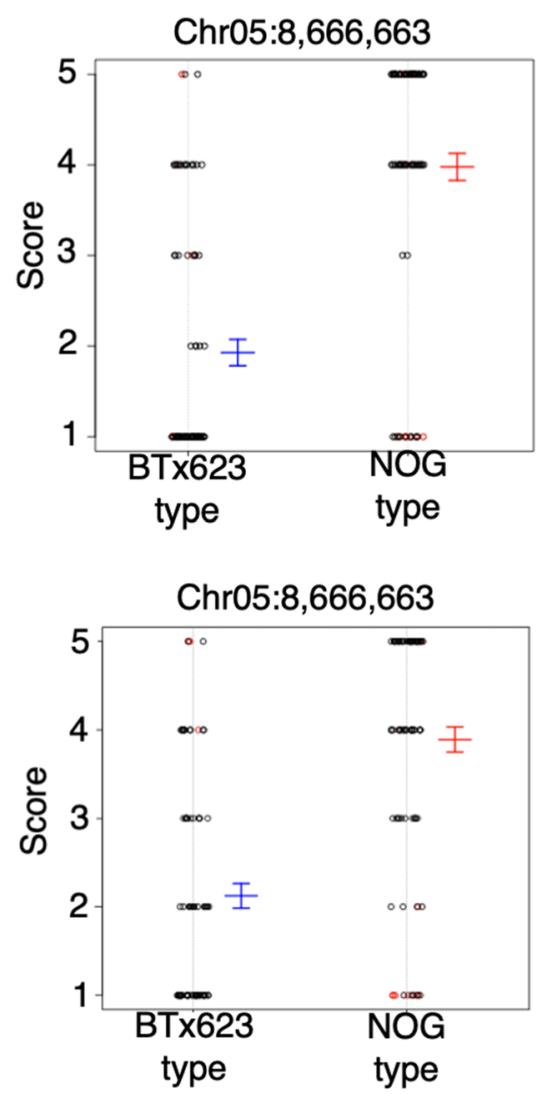

b

\begin{tabular}{lccccccc}
\hline QTL & Generation Chr $\begin{array}{c}\text { Marker interval } \\
(1.5-\text { LOD })\end{array}$ & $\begin{array}{c}\text { Nearest } \\
\text { marker }\end{array}$ & $\begin{array}{c}\text { Position } \\
(\mathrm{cM})\end{array}$ & LOD & $\begin{array}{c}\text { PVE } \\
(\%)\end{array}$ \\
\hline qOPS5 & $\mathrm{F}_{9}$ & 5 & $\begin{array}{l}\text { Chr05.7,756,251- } \\
\text { Chr05.8,912,816 }\end{array}$ & Chr05:8,666,663 & 22.1 & 21.9 & 42.3 \\
qOPS5 & $\mathrm{F}_{10}$ & 5 & $\begin{array}{l}\text { Chr05.7,903,844 } \\
- \text { Chr05.8,768,110 }\end{array}$ & Chr05:8,666,663 & 22.1 & 26.4 & 52.6 \\
qOPS5 & $\mathrm{F}_{11}$ & 5 & $\begin{array}{l}\text { Chr05.6,767,884 } \\
- \text {-Chr05.8,912,816 }\end{array}$ & Chr05:8,666,663 & 22.1 & 9.9 & 18.5 \\
\hline
\end{tabular}

Figure 2. QTL analysis of OPS phenotype with fenitrothion treatment in the $\mathrm{F}_{9}, \mathrm{~F}_{10}$, and $\mathrm{F}_{11}$ RIL populations. (a) The logarithm of odds (LOD) graphs for fenitrothion treatment using RILs in each population (left). The gray line represents an LOD threshold $=3$, and the red line represents an LOD threshold based on a permutation test with 1000 iterations. Right panel represents plot phenotypes versus marker genotypes analysis corresponding to graphs. (b) Summary of QTLs identified in (a). The same QTL marker was obtained in the $\mathrm{F}_{9}, \mathrm{~F}_{10}$, and $\mathrm{F}_{11}$ RIL generations. PVE indicates phenotype variation explained. Marker intervals were estimated based on confidence intervals (1.5-LOD). 
weak signals in all samples, and the expression level did not respond to fenitrothion treatment. In contrast, $N L R-C$ was shown to be expressed at a substantial level and to be detectable by our RT-PCR analyses ( 30 and 35 cycles). Intriguingly, $N L R-C$ was constitutively expressed in NOG regardless of fenitrothion treatment, whereas no signals corresponding to $N L R-C$ transcripts were detected in BTx623. Consistent with this observation, RIL25 $\mathrm{F}_{9}$ segregants heterozygous for OSR and exhibiting OPS phenotype accumulated NLR-C mRNAs, whereas segregants homozygous for $O S R$ and exhibiting resistance to fenitrothion was missing $N L R-C$ transcripts (Fig. 6a).

Functionality of NLR-C in NOG was accounted for by the presence of the promoter. To account for the differential expression of $N L R-C$, we reasoned that the 593-bp insertion present in NOG may positively affect transcription. This insertion is located $138 \mathrm{bp}$ upstream of the ATG initiation codon of NLR-C extending towards the $5^{\prime}$ direction (Fig. 6b), suggesting that this insertion generates transcription initiation and/or part of $5^{\prime}$-untranslated region (UTR). Given that no transcript accumulated in BTx623, we postulated that the insertion contains a promoter activity and transcription initiates from this region. To examine this possibility, $5^{\prime}$-rapid amplification of cDNA ends (RACE) was performed with total RNA extracted from NOG and BTx623 with specific primers to detect the $5^{\prime}$ end of $N L R-C$ transcripts (Supplementary Fig. S5a). The result showed that, as expected, the amplified band corresponding to the $5^{\prime}$ end was detectable only in NOG (Supplementary Fig. S5b). Sequencing of the amplified fragment demonstrated that the $5^{\prime}$ end lies in A at 365-bp upstream of ATG initiation codon (Supplementary Fig. S5c). Consequently NLR-C mRNA possesses a 365-bp 5'-UTR, within which no additional ORF was found. Furthermore, searches for core promoter elements in O. sativa in the database (http://bioinformatics.psb.ugent.be/webtools/plantcare/html) revealed that a putative promoter sequence, TAT ATAACAATATATGTACAAAA, exists 162-bp upstream of the start-site of $N L R-C$ transcripts, which is also included in the 593-bp insertion (Fig. 6). Based on these results, we concluded that BTx623 has a null mutation in NLR-C due to the loss of a functional promoter, and that NLR-C is responsible for OPS in NOG.

\section{Discussion}

Sensitivity to organophosphate pesticides has been reported in sorghum by several research groups, as organophosphate insecticide reaction (opr $)^{42,43}$ and resistance to chemical burning $(r c b)^{45}$. Although they mapped these loci using conventional DNA markers with different mapping populations, their location on chromosome 5 was suggested to represent the same locus ${ }^{44}$. Later, Boyles et al. (2017) characterized a 'crop injury' QTL as correlated with $o p r$; the corresponding chromosomal region on chromosome 5 was shown to have an NB-LRR gene cluster, although no further experimental verification was provided ${ }^{24}$. More recently, Kawahigashi et al. (2020) investigated organophosphate-sensitive reaction (osr) using a sensitive inbred Nakei MS3B and raised the possibility that the same $N B-L R R$ gene cluster is indeed responsible for $\mathrm{OPS}^{46}$. Based on the differential accumulation of transcripts in these three genes (termed $A, B$, and $C$ in this study), $N L R-C$ was supposed to be the major gene controlling OPS. The existence of amino-acid substitutions between Nakei MS3B and the resistant lines (BTx623 and Greenleaf) was also reported for NLR-C. Our current mapping of OPS observed in NOG was consistent with these previous reports. Detailed comparison of the nucleotide sequence along with transcript accumulation between BTx623 and NOG led us to conclude that NLR-C is the major gene.

Although these reports identified $N L R-C$ as a candidate gene, a discrepancy exists over the dominance of OPS. In most of studies, OPS was shown to be dominant. However, only Kawahigashi et al. (2020) reported OPS in Nakei MS3B as recessive ${ }^{46}$. As for the expression of NLR-C, they showed that resistant BTx623 had no detectable transcripts, whereas sensitive Nakei MS3B had substantial levels of $N L R-C$ transcripts, strongly arguing against the dominant role of resistance in BTx623. In fact, we demonstrated here that BTx623 has a null mutation due to the loss of promoter activity, which cannot cause a dominant effect on resistance. One possibility to explain this inconsistency may be the unstable appearance of OPS in the heterozygotes. In our study, a careful inspection of $F_{1}$ plants showed that the lesion owing to OPS was weak and observed unstably at the seedling stage, whereas OPS became rather apparent in mature leaves (Fig. 4). These observations implied that the expression of OPS may be influenced developmentally, although the precise reason for this instability was unclear. We thus consider that re-evaluation is necessary to confirm whether OPS in Nakei MS3B is dominant.

Given the dominant effect of OPS in NOG, we further characterized the NB- $L R R$ gene cluster. We excluded $N L R-A$ because it is missing in NOG. Therefore, we considered that $N L R-B$ and $N L R-C$ are responsible for OPS. As for $N L R-B$, transcripts accumulated both in NOG and BTx623, whereas two amino acid substitutions in NOG (I289V and S650R) resides in the NB and LRR domains, respectively, and may account for the dominant effect of OPS. As for NLR-C, we have presented compelling evidence that the 593-bp deletion in BTx623 leads to the loss of promoter activity and a null mutation. Thus, a 593-bp insertion in the promoter region of $N L R-C$ may be a determinant of the OPS phenotype in NOG. To test if NLR-C is involved and has dominant role in OPS, we performed heterologous complementation assays using agrobacterium infiltration in Nicotiana benthamiana (Supplementary Fig. S6). Overexpression of NLR-C and concomitant treatment with fenitrothion, however, did not result in necrotic lesions, as opposed to the control, suggesting that OPS is not simply conferred by NB-LRR and may require species-specific signaling cascades to respond to organophosphate pesticides. An alternative possibility is the requirement of both $N L R-B$ and $N L R-C$ to drive OPS.

OPS has been extensively characterized in tomato $25,27,50$. The Fen gene, which corresponds to the organophosphate pesticide fenitrothion sensitivity phenotypes, encodes a serine-threonine protein kinase ${ }^{28}$. The Fen and Pto genes share $87 \%$ amino acid similarity. Fen kinase is needed for fenthion sensitivity. Reports have shown that Fen stimulates organophosphate pesticide fenthion-inducible signaling and interacts with the N-terminal domain of a NB-LRR protein $\operatorname{Prf}^{29}$. Prf is indispensable for fenthion sensitivity, but the role of Fen and its association with 
Figure 3. Three NB-LRR genes (NLR-A, $N L R-B$, and $N L R-C)$ located in the OSR locus. (a) Graphical genotypes of RIL lines showing recombination at the putative OSR region on chromosome 5. OSR was mapped within a 743-kb region between SNP markers Chr05:7,971,429 and Chr05:8,714,734. The gray, striped, and black bars represent chromosomal regions homozygous for BTx623, heterozygous, and homozygous for NOG, respectively. ' $\mathrm{R}$ ' and ' $\mathrm{S}$ ' indicates lines resistant and sensitive to fenitrothion, respectively. (b) A closeup view of the putative OSR locus, located near SNP marker Chr05:8,666,663. Gray, red, blue, and green arrows indicate the positions of the UDP-glucoronosyl transferase gene, $N L R-A, N L R-B$, and $N L R-C$, respectively. The smaller black arrows indicate the relative location of primers used to amplify $N L R-A$ in (c). The dashed line indicates the missing region from 8,457,439 to 8,557,269 including NLR-A in NOG. The green arrowhead indicates the 593-bp insertion upstream of NLR-C in NOG. (c) Agarose gel electrophoresis of PCR products corresponding to NLR$A$, showing that NOG lacks this gene. The original image of the gel is provided in Supplementary Information 2. (d) Schematic diagrams showing the presumed coiled-coil (CC), nucleotide binding site (NB), and leucine-rich repeat (LRR) domains in NLR-B (upper) and NLR-C (lower) proteins. Predicted amino acid substitutions in NOG, compared with those in BTx623, are indicated.

$\operatorname{Prf}^{51}$ in fenthion sensitivity are poorly understood. Another example of an NB-LRR responding to chemicals is At5g46520 in Arabidopsis, which responds to accession-specific root growth arrest with DFPM ${ }^{32,33}$. DFPM was shown to be related to several abscisic acid (ABA) responses, including rapid disruption of ABA-induced stomatal closing and ABA activation of guard cell anion channels. Detailed analyses have uncovered that DFPM stimulates an ETI and results in rapid disruption of ABA signal transduction. A genetic variant of the NB-LRR gene in the Arabidopsis Columbia-0 accession is responsive specifically to DFPM by generating primary root meristem arrest.

Although there has been extensive research on $\mathrm{R}$ genes that defense against microbial pathogens, there are few studies on the toxic reaction in plants from organophosphate chemicals. In sorghum, the resistance to a necrotrophic fungus Bipolaris sorghicola, controlled by $d s 1$ encoding an NB-LRR has been reported ${ }^{52}$. Because $O S R$ and $d s 1$ are distantly mapped on chromosome 5 , the OSR locus is unlikely to be involved in the resistance to $B$. sorghicola ${ }^{53}$. Future study is required to test if any of the NLR genes in the OSR locus is associated with pathogens, similarly to Pto/Fen in tomato. NLR-C shows similarity to the NB-LRR protein Arabidopsis RPM1 (Supplementary Fig. S4). Notably, a mutation in one of the rice RPM1 homologs OsRLR1 resulting from ethyl methane sulfonate treatment led to spontaneous hypersensitive response-like lesions with strong growth reduction, which was similar to the OPS phenotype ${ }^{48}$. Based on the results in Supplementary Fig. S5, it appears that NLR-C indirectly recognizes fenitrothion. RPM1 indirectly recognizes the Pseudomonas syringae effectors AvrB and AvrRpm1 through an Arabidopsis RIN4 protein ${ }^{54}$. AvrB and AvrRpm1 phosphorylate RIN4, and RPM1 monitors this phosphorylation and causes ETI. It is possible that NLR-C employs a host protein(s) to sense fenitrothion. Alternatively, evidence has been accumulating that sensor and helper NB-LRR pairs work together to perceive pathogen effectors and induce $\mathrm{ETI}^{55}$. Conceptually, it is possible that NLR-B and NLR-C act in a complex with the organophosphate molecules or act downstream as part of the OSR signaling pathway.

Our previous analysis of QTLs related to biomass in the same population did not find a QTL on chromosome 5. Therefore, its impact on biomass is unlikely. However, the OPS phenotype might decrease production in sorghum upon pesticide application, and our understanding of the OSR locus presented in this study is important in future sorghum breeding. In particular, unstable appearance of OPS along with its dominance over resistance should be specially taken into consideration because it potentially affects $F_{1}$ hybrids commonly used for commercial sorghum production. Our preliminary evaluation of OPS among the sorghum core collection used in our previous study indicated that most sorghum inbred lines are free from OPS. Nevertheless, two additional lines (IS11331 and Manfredi Cholila) were shown to have the same OSR haplotype as NOG and exhibit OPS at the seedling stage (Supplementary Fig. S7). Our previous admixture analysis categorized the inbred lines into three groups, where NOG belonged to Group II that represented Asian accessions ${ }^{39}$. While both NOG and Manfredi Cholila were clustered in Group II, IS11331 belonged to Group I. Although further study is required, these results may implicate that the rare OPS originate from multiple genetic backgrounds.

In conclusion, the presented results along with previous reports strongly suggest that the OSR locus corresponds to the NB-LRR gene cluster, particularly to NLR-B and NLR-C. OSR is dominant with the unstable appearance of OPS in the $F_{1}$ generation, implicating particular caution in securing proper leaf longevity in breeding programs. Further investigations should allow us to understand the occurrence of OPS and its generic variation among sorghum cultivars, which may be related to diversification of the $N B-L R R$ gene families.

\section{Methods}

Plant materials. Two parental lines, BTx623 and NOG, and the RIL population were established as described in our previous study ${ }^{39}$. All plant materials including each RIL generation were grown, harvested, and stored at the Institute of Plant Science and Resources (IPSR), according to the institutional guideline to handle plant materials. A genetic map was constructed using RAD-seq, and the genotyping data of 213 individuals were constructed in the $\mathrm{F}_{6}$ population. In this study, the $\mathrm{F}_{9}, \mathrm{~F}_{10}$, and $\mathrm{F}_{11}$ populations were subjected to phenotyping of pesticide resistance, followed by QTL analysis. Phenotyping was carried out in multiple years with one replicate for each cultivation. Plants were germinated in small trays filled with vermiculite for approximately 10-14 days, subsequently transplanted to $30-\mathrm{cm}$ diameter pots filled with soil from IPSR field. A single RIL line (RIL25) segregating for OPS was obtained by screening the $\mathrm{F}_{8}$ population. Seeds from RIL25 were harvested from an $\mathrm{F}_{8}$ individual that was heterozygous to OSR (confirmed by PCR), and the resulting $\mathrm{F}_{9}$ segregants were subjected to segregation analysis. $F_{1}$ seeds were generated by crossing NOG with BTx623 with manual emasculation. 
a

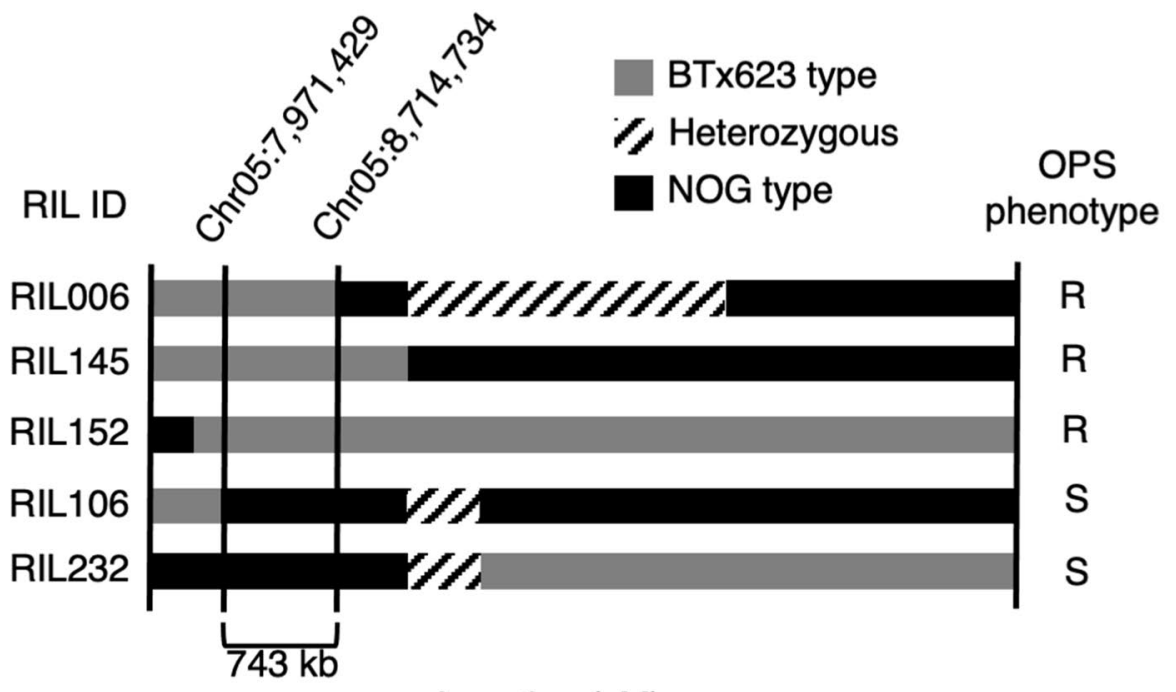

b

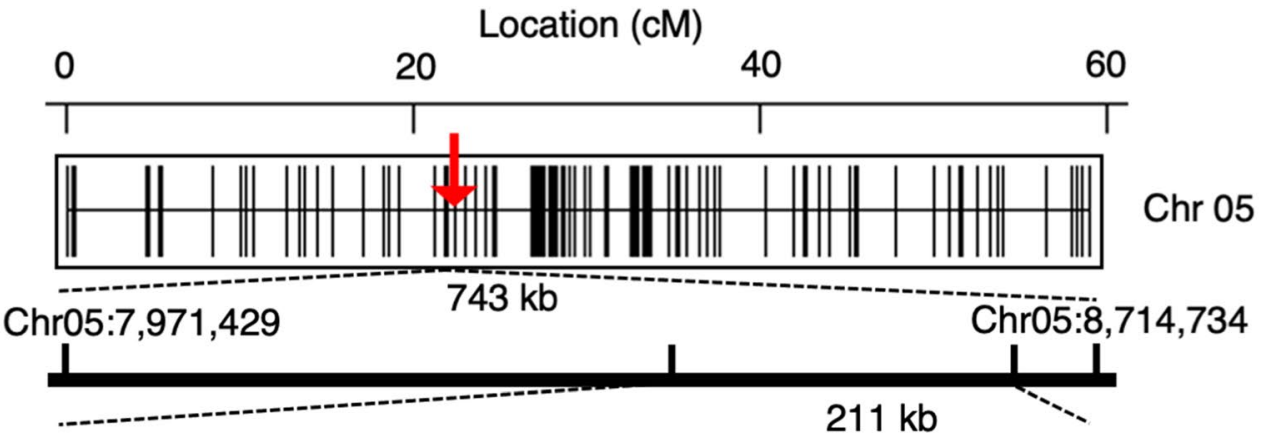

Chr05:8,455,553

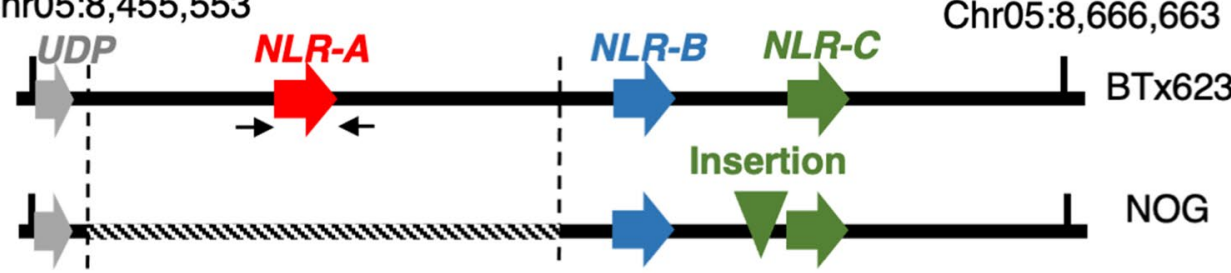

Chr05:8,457,439

Chr05:8,557,269

C

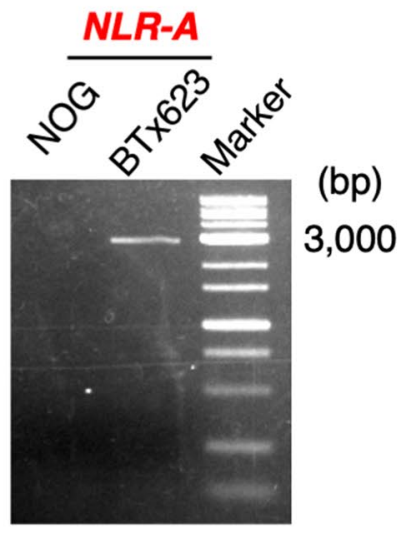

d

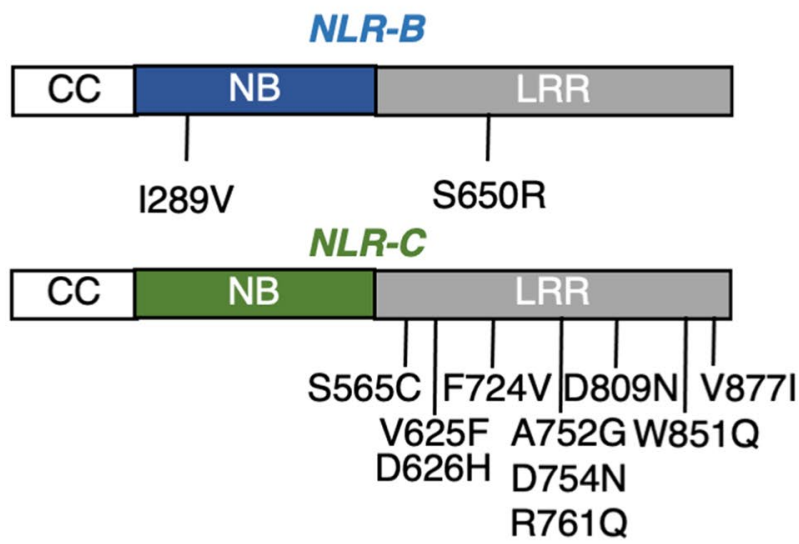




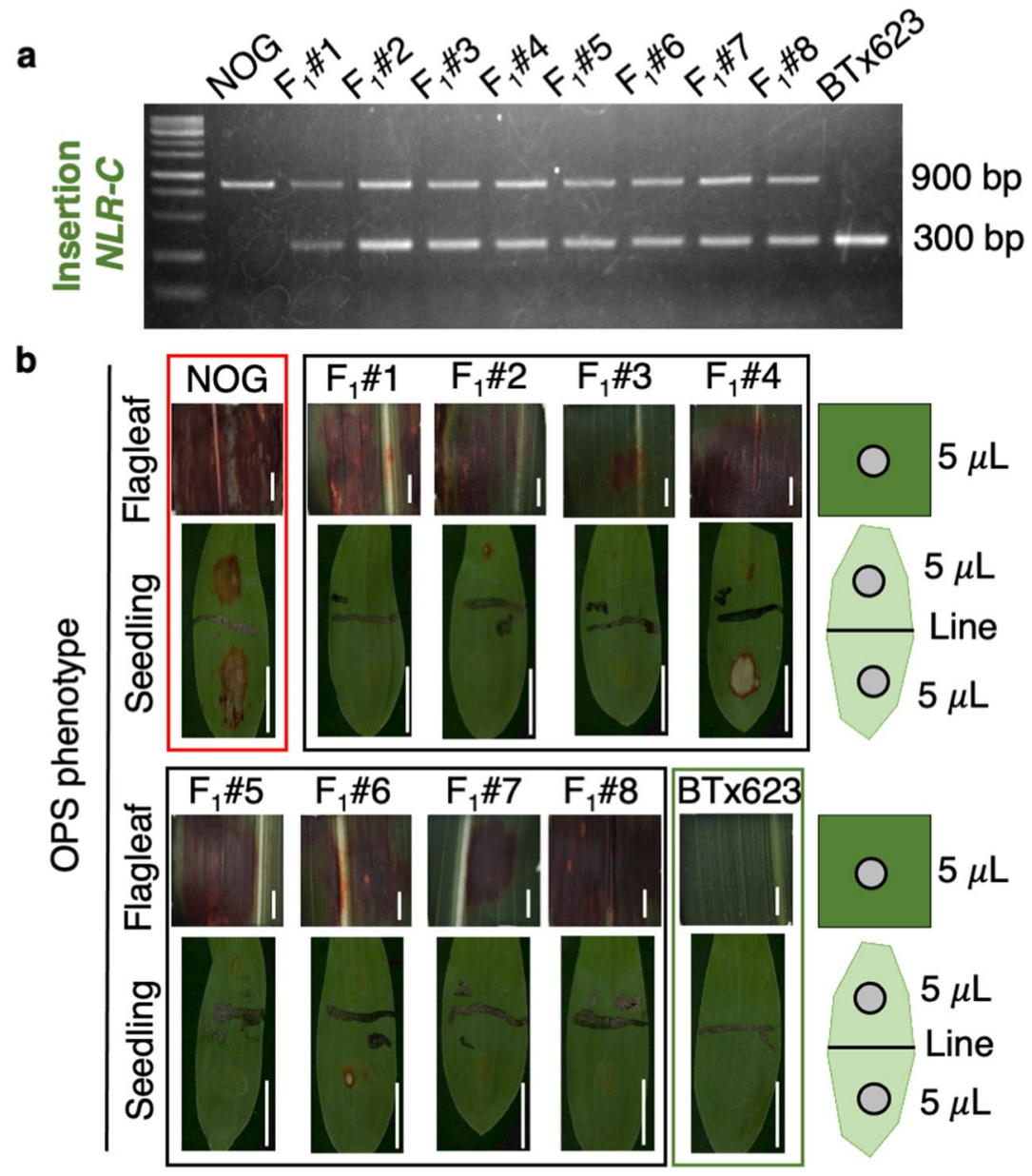

Figure 4. Semi-dominant OPS phenotype confirmed in $\mathrm{F}_{1}$ individuals. (a) Heterozygosity was confirmed by genotyping $N L R-C$ with a pair of specific primers (NLR-C_Insertion_F and NLR-C_Insertion_R) that distinguish NOG-specific insertion (900 bp in NOG). The original image of the gel is provided in Supplementary Information 2. (b) $\mathrm{F}_{1}$ plants (\#1-\#8) were grown from seedling to mature stages along with NOG and BTx623, and fenitrothion was spotted onto the fourth or fifth leaves at the seedling stage as illustrated (lower panels. Scale bars; $1 \mathrm{~cm}$ ) or sprayed onto mature leaves (upper panels, Scale bars; $0.6 \mathrm{~cm}$ ).

Phenotyping OPS with organophosphate pesticide treatment. The trials were performed in an open-air greenhouse at the experimental field of IPSR (latitude: $34^{\circ} 35^{\prime} 31^{\prime \prime} \mathrm{N}$, longitude: $133^{\circ} 46^{\prime} 7^{\prime \prime} \mathrm{E}$ ), Kurashiki city, Okayama, Japan. Seedlings at the fourth-leaf stage were sprayed uniformly with $500 \times$ diluted fenitrothion, malathion or acephate (Sumitomo Chemical Garden Products INC, Japan), and the phenotype was scored visually (from 1 to 5 ) at 7 days after the pesticide treatment. A score of 1 indicated essentially no leaf death, a score of 3 indicated approximately $50 \%$ of the leaf area was dead, and a score of 5 indicated $100 \%$ seedling (leaves and stem) death. The sensitive lines showed red-brown spots and necrotic lesions throughout their growth, and the resistant lines maintained greener leaf area. Flag leaves were treated with fenitrothion from $\mathrm{F}_{1}$ plants and the RIL25- $\mathrm{F}_{9}$ segregating population at the heading stage, and the greenness of plants was estimated visually 7 days after fenitrothion application. Moreover, evaluation of the greenness trait with fenitrothion treatment was performed for each RIL.

QTL analysis. Genotype data obtained from $\mathrm{RAD}$-seq in the $\mathrm{F}_{6}$ population was used to perform QTL analysis in this study. A total of 3710 SNPs distributed across the genome were obtained. A dense linkage map with 3710 markers spanning a distance of $644.8 \mathrm{cM}$ on all chromosomes, with an average and maximum spacing between markers of $0.2 \mathrm{cM}$ and $7.1 \mathrm{cM}$, respectively, was developed previously. Genotype probabilities were calculated using the calc.genoprobability function with a step size of $1 \mathrm{cM}$ and an assumed genotyping error probability of 0.05 using the Kosambi map function as implemented in the R/qtl package ${ }^{56,57}$. QTL analysis was performed using the CIM function of the R/qtl package with the Haley-Knott regression method $^{58}$. Linkage analysis was performed using the R/qtl package in $\mathrm{R}$ version 3.4.2. The LOD significance threshold for detecting QTLs was calculated by performing 1000 iterations using the R/qtl permutation test. 
a The OPS phenotype

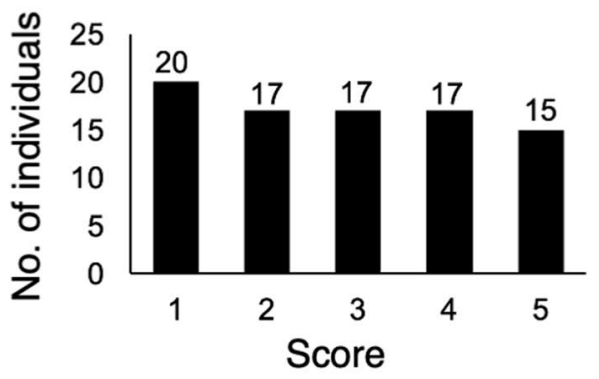

b

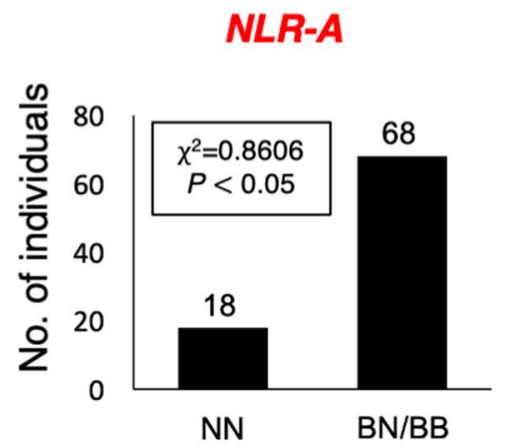

C

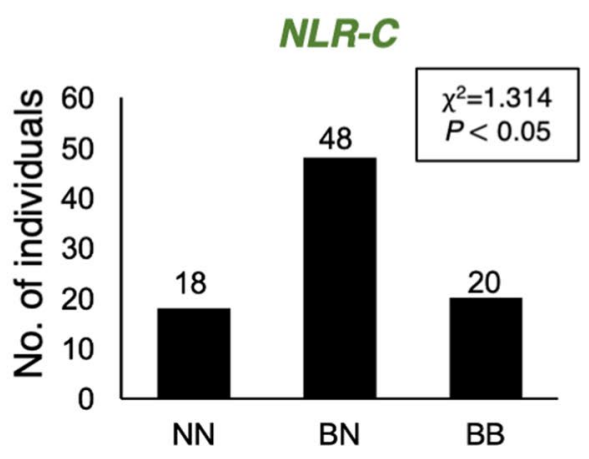

d
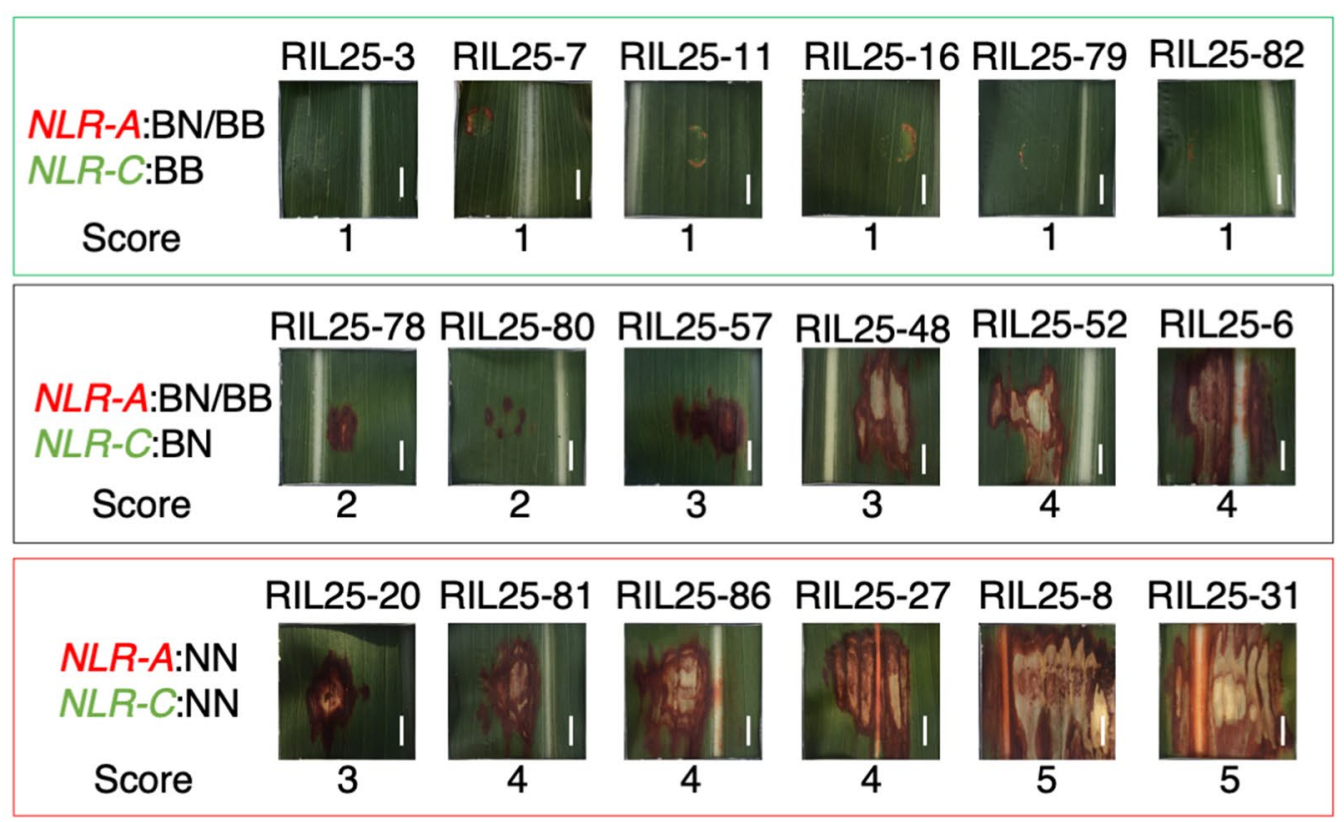

Figure 5. Linkage analysis of OPS with NLR-C in the RIL25- $\mathrm{F}_{9}$ segregating population. (a) Histogram of 86 individuals subjected to segregation analysis. The $\mathrm{Y}$ axis represents numbers of individuals and the $\mathrm{X}$ axis represents the scores, a score of 1 indicates resistance to OP treatment, whereas a score of 5 indicates sensitivity. (b) Segregation of $N L R-A$ genotypes among $\mathrm{F}_{9}$ individuals showing a 1:3 ratio $\left(P<0.05, \chi^{2}=0.8606\right)$. (c) Segregation of NLR-C genotypes among $\mathrm{F}_{9}$ individuals showing a 1:2:1 ratio $\left(P<0.05, \chi^{2}=1.314\right)$. (d) Representative images of OPS phenotypes in the corresponding genotypes. The genotype of the OSR locus is indicated on the left, and the OPS phenotypes from individuals homozygous for BTx623 (top), heterozygous (middle), and homozygous for NOG (bottom) are shown. Scale bars; $0.6 \mathrm{~cm}$. 
Figure 6. Expression of NLR genes in OSP and null mutation in NLR-C. (a) Transcript accumulation of the three NB-LRR genes ( $N L R-A, N L R-B$, and $N L R-C)$ estimated using semi-quantitative RT-PCR. Expression of $N L R-A, N L R-B$, and $N L R-C$ in leaves. Accumulation of $N L R-C$ transcripts coincides with the presence of the fenitrothion-sensitive (S) NOG genotype (NOG, RIL25-78,-80, and-81), whereas no transcript was detected in BTx623 and the resistant (R) line (RIL25-79). ' + ' and '-' indicate gene expression analysis in the presence or absence of organophosphate pesticide (OP), respectively. The PP2A gene was used as an internal control. The original gel images are provided in Supplementary Information 2. (b) Schematic diagram of the NLR-C coding region and its 5 ' upstream region. NLR-C consists of a single exon (top), and the closeup view of the 593-bp insertion specific to NOG is shown below. Putative transcription start site (TSS, indicated by an arrow) is located 227 bp upstream from the $3^{\prime}$ end of the insertion. The nucleotide sequence of the insertion is shown below the diagram, where the putative TSS determined by $5^{\prime}$ RACE and the TATA-box sequence are indicated. A schematic diagram of the insertion region is shown, which contains the TSS and putative TATA-box, upstream $N L R-C$ gene in NOG genetic background.

Phylogenetic analysis of OSR genes. A phylogenetic tree using amino acid alignment of the three NBLRR proteins (NLR-A, NLR-B, and NLR-C) and other typical R proteins from other species was constructed using a neighbor-joining tree ${ }^{59}$. The evolutionary distances were computed using the Poisson correction method ${ }^{60}$ and are in units of the number of amino acid substitutions per site. The R proteins used for phylogenetic analysis were as follows: S. bicolor, Sobic.005G071700 (NLR-A), Sobic.005G071900 (NLR-B), Sobic.005G072000 (NLR-C), Sobic.008G117000, Sobic.005G070500 and Sobic.001G291000. O. sativa, LOC_Os10g07978 (OsRLR1) ${ }^{48}$, LOC_ Os06g22460 (Pid3) ${ }^{49}$, LOC_Os01g36640, LOC_Os10g07534 and LOC_Os04g46300. A. thaliana, AT3G07040 $(\mathrm{RPM} 1)^{47}$. Phylogenetic analyses were performed using MEGA $\mathrm{X}^{61}$.

Cloning of the OSR genes. The coding regions of the NLR-B and NLR-C genes were amplified by PCR using KOD FX Neo polymerase (TOYOBO, Japan) with specific primer pairs, InFusion_NLR-B_Xho1_fw and InFusion_NLR-B_Not1_rv, InFusion_NLR-C_EcoR1_fw and InFusion_NLR-C_BamH1_rv, respectively. The program involved: an initial denaturation step at $94{ }^{\circ} \mathrm{C}$ for $2 \mathrm{~min}, 35 \mathrm{cycles}$ of denaturation step at $98{ }^{\circ} \mathrm{C}$ for $10 \mathrm{~s}$, annealing step at $60^{\circ} \mathrm{C}$ for $30 \mathrm{~s}$ and extension step at $72{ }^{\circ} \mathrm{C}$ or $5 \mathrm{~min}$, and a final extension at $72{ }^{\circ} \mathrm{C}$ for $10 \mathrm{~min}$. The PCR fragment of $N L R-B$ was cloned using an In-Fusion HD cloning kit (Takara, Japan), into pGreen0029_35S digested and linearized with Xho1 and Not1. Likewise, the PCR fragment of NLR-C was cloned into pGreen0029_35S digested with the EcoR1 and BamH1. Deletion of NLR-A in NOG was examined by PCR amplification of genomic DNA templates using KOD FX Neo polymerase (TOYOBO, Japan).

Gene expression analysis. Flag leaves at the heading stage were used for expression analysis of NLR-A, $N L R-B$, and $N L R-C$. Total RNA was extracted from plant samples using an RNeasy Plant Mini Kit (Qiagen, Germany). First-strand cDNA was synthesized from $500 \mathrm{ng}$ of each RNA sample in a $50 \mu \mathrm{L}$ reaction solution using the SuperScript III First-Strand System (Thermo Fisher Scientific, USA). PCR was performed using $1 \mu \mathrm{L}$ aliquots of cDNA solution in a $25-\mu \mathrm{L}$ volume with KOD FX Neo DNA polymerase (TOYOBO, Japan). The PCR profile was as follows: initial denaturation for $2 \mathrm{~min}$ at $94^{\circ} \mathrm{C} 28-35$ cycles of $15 \mathrm{~s}$ at $94{ }^{\circ} \mathrm{C} 30 \mathrm{~s}$ at $60^{\circ} \mathrm{C}$ and $1 \mathrm{~min}$ at $68^{\circ} \mathrm{C}$ then $5 \mathrm{~min}$ at $68^{\circ} \mathrm{C}$ or final extension. Five microliter aliquots of the PCR products were analyzed by electrophoresis in $1 \%$ agarose gels. Primers used in this study are listed in Supplementary Table S2.

5'-RACE analysis of candidate gene. The 5' RACE experiment was performed using a 5-Full RACE Core Set Kit (TAKARA, Japan) in accordance with the manufacturer's instructions. Fifteen microliters of ligated cDNA was then used as a template for nested PCR using KOD FX Neo polymerase (TOYOBO, Japan) with two sets of primers, the outside set was sense primer_1 and antisense primer_1, and the inside set is sense primer_2 and antisense primer_2. The PCR reaction mixture was incubated for $2 \mathrm{~min}$ at $94^{\circ} \mathrm{C}$ followed by 30 amplification cycles, comprising denaturation at $94^{\circ} \mathrm{C}$ for $30 \mathrm{~s}$, annealing at $60^{\circ} \mathrm{C}$ for $30 \mathrm{~s}$ and extension at $68^{\circ} \mathrm{C}$ for $30 \mathrm{~s}$. The reaction was extended for another $10 \mathrm{~min}$ at $68^{\circ} \mathrm{C}$ to ensure full extension. Primers used in this study are listed in Supplementary Table S2.

Transient expression of NLR-C in Nicotiana benthamiana. Agroinfiltration of N. benthamiana was performed as described previously ${ }^{62}$. A. tumefaciens strain GV3101, carrying NLR-C, Pit D485V, or GFP was used to infiltrate leaves of 5-week-old $N$. benthamiana plants. p19 silencing suppressor was used to enhance gene expression. Each agrobacterium culture was resuspended in buffer containing $10 \mathrm{mM} \mathrm{MgCl}, 10 \mathrm{mM} \mathrm{MES}, \mathrm{pH}$ 5.6, and $150 \mu \mathrm{M}$ acetosyringone, and incubated at room temperature for $2-3 \mathrm{~h}$ before infiltration. After incubation for $24 \mathrm{~h}$, fenitrothion (1:7500 dilution) or distilled water were infiltrated into the same spots as used for agroinfiltration. The photosynthetic capacity was measured at 3 days post-inoculation (dpi) using an imaging fluorimeter (FluorCam $800 \mathrm{MF}$, photon systems instruments). Infiltrated plants were kept at $22^{\circ} \mathrm{C}$. Photographs were taken at 17 days post-inoculation (dpi) for the assessment of cell death. 
a

\begin{tabular}{|c|c|c|c|c|c|c|}
\hline $\mathrm{R} / \mathrm{S}$ & $S$ & R & S & R & $\mathrm{S}$ & $\mathrm{S}$ \\
\hline Line & NOG & BTx623 & RIL25-78 & RIL25-79 & RIL25-80 & RIL25-81 \\
\hline OP & $-\quad+$ & $-\quad+$ & $-\quad+$ & + & + & - \\
\hline
\end{tabular}

NLR-A

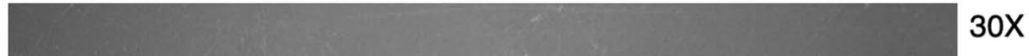

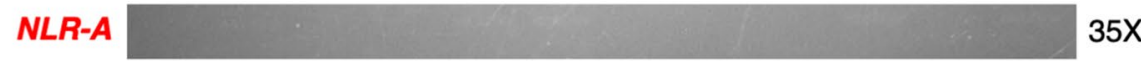

$\begin{array}{lll}N L R-B & 30 X\end{array}$

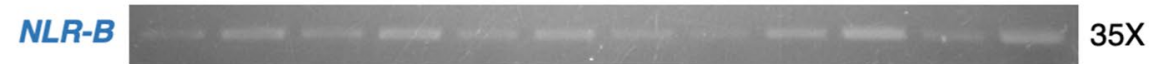

NLR-C

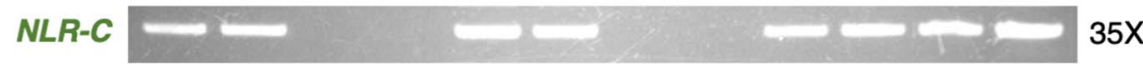

\begin{tabular}{l|l|l|} 
PP2A & $28 \mathrm{X}$
\end{tabular}

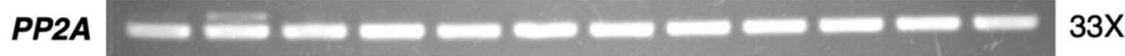

b

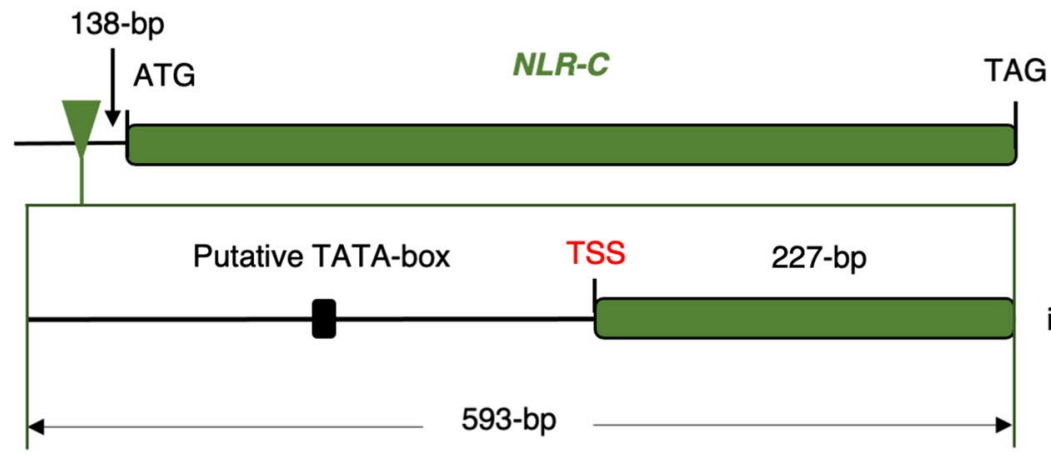

in NOG

5'-AAAAAGCAAAATGTTTTTTTTTCCTTCATCGTTCATATGTCCGATGACACC

TCTTGCACACACGTCCACTTGCAACTCTAGACACCTGAGTTAGACCGATCGT

GTTCAAAACCGTTCAAGCTGTCTGATACTGCCCCTAGTACTACATCTCACAT Putative TATA-box

TTGTGAATAAACAACATGATCGACAATATATAACAATATATGTACAAAATGTC

TATTAAAGTATACAGTAATTCTGGTCACCTGCTCCCACAATTAAACAAAGCTT

TCCCCTTTTTCTCACTCCTTCCTATGCTGCTGGTAGCGTTACTATCCAGGTA

CACAATCAGCTTGGTCCTTTCTTCCACAATGACGAAGGCACAAGCCCACAAC $\longrightarrow$ TSS

CACTTCATGTTCGCCGTTGACTCGTCGAGTTGTGGTCGCTGTTCTAGGGCC

TTGTAGCATGGTTTCTTAGTTCTTACAGGATGCAAATCCAGATCTCCTACTC

CGCCGAAGTTGACAGTGGCTTCAGCATGGCCGGAAGCCCAGATCTGCAG

CGCGTGCTTGTTTTCTAGCACCTCGTTTTCAGGTATATATAAGATCAGATCT

CGTCAGTTCACAATTTGTTCAATG - 3' 
Received: 6 May 2021; Accepted: 14 September 2021

Published online: 06 October 2021

\section{References}

1. Costes, E., Lauri, P. E., Simon, S. \& Andrieu, B. Plant architecture, its diversity and manipulation in agronomic conditions, in relation with pest and pathogen attacks. Eur. J. Plant Pathol. 135, 455-470. https://doi.org/10.1007/s10658-012-0158-3 (2013).

2. Wheelis, M., Casagrande, R. \& Madden, L. V. Biological attack on agriculture: Low-tech, high-impact bioterrorism. Bioscience 52, 569-576. https://doi.org/10.1641/0006-3568(2002)052[0569:Baoalt]2.0.Co;2 (2002).

3. Grieve, B. D. et al. The challenges posed by global broadacre crops in delivering smart agri-robotic solutions: A fundamental rethink is required. Glob. Food Secur. Agric. 23, 116-124. https://doi.org/10.1016/j.gfs.2019.04.011 (2019).

4. Bisht, D. S., Bhatia, V. \& Bhattacharya, R. Improving plant-resistance to insect-pests and pathogens: The new opportunities through targeted genome editing. Semin. Cell Dev. Biol. 96, 65-76. https://doi.org/10.1016/j.semcdb.2019.04.008 (2019).

5. Fischer, R. A. T. \& Edmeades, G. O. Breeding and cereal yield progress. Crop Sci. 50, S85-S98. https://doi.org/10.2135/cropsci2009. 10.0564 (2010).

6. Balint-Kurti, P. The plant hypersensitive response: Concepts, control and consequences. Mol. Plant Pathol. 20, 1163-1178. https:// doi.org/10.1111/mpp.12821 (2019).

7. Liu, J., Liu, X., Dai, L. \& Wang, G. Recent progress in elucidating the structure, function and evolution of disease resistance genes in plants. J. Genet. Genomics 34, 765-776. https://doi.org/10.1016/S1673-8527(07)60087-3 (2007).

8. Li, X., Kapos, P. \& Zhang, Y. NLRs in plants. Curr. Opin. Immunol. 32, 114-121. https://doi.org/10.1016/j.coi.2015.01.014 (2015).

9. Cui, H., Tsuda, K. \& Parker, J. E. Effector-triggered immunity: From pathogen perception to robust defense. Annu. Rev. Plant Biol. 66, 487-511. https://doi.org/10.1146/annurev-arplant-050213-040012 (2015).

10. Marone, D., Russo, M. A., Laido, G., De Leonardis, A. M. \& Mastrangelo, A. M. Plant nucleotide binding site-leucine-rich repeat (NBS-LRR) genes: Active guardians in host defense responses. Int. J. Mol. Sci. 14, 7302-7326. https://doi.org/10.3390/ijms140473 02 (2013).

11. Kourelis, J. \& van der Hoorn, R. A. L. Defended to the nines: 25 years of resistance gene cloning identifies nine mechanisms for $\mathrm{R}$ protein function. Plant Cell 30, 285-299. https://doi.org/10.1105/tpc.17.00579 (2018).

12. Collier, S. M. \& Moffett, P. NB-LRRs work a "bait and switch" on pathogens. Trends Plant Sci. 14, 521-529. https://doi.org/10. 1016/j.tplants.2009.08.001 (2009).

13. Collier, S. M., Hamel, L. P. \& Moffett, P. Cell death mediated by the N-terminal domains of a unique and highly conserved class of NB-LRR protein. Mol. Plant Microbe Interact. 24, 918-931. https://doi.org/10.1094/Mpmi-03-11-0050 (2011).

14. Swiderski, M. R., Birker, D. \& Jones, J. D. G. The TIR domain of TIR-NB-LRR resistance proteins is a signaling domain involved in cell death induction. Mol. Plant Microbe Interact. 22, 157-165. https://doi.org/10.1094/Mpmi-22-2-0157 (2009).

15. Zhu, X. L. et al. The wheat NB-LRR gene TaRCR1 is required for host defence response to the necrotrophic fungal pathogen Rhizoctonia cerealis. Plant Biotechnol. J. 15, 674-687. https://doi.org/10.1111/pbi.12665 (2017).

16. Igari, K. et al. Constitutive activation of a CC-NB-LRR protein alters morphogenesis through the cytokinin pathway in Arabidopsis. Plant J. 55, 14-27. https://doi.org/10.1111/j.1365-313X.2008.03466.x (2008).

17. Perry, E. D., Ciliberto, F., Hennessy, D. A. \& Moschini, G. Genetically engineered crops and pesticide use in U.S. maize and soybeans. Sci. Adv. 2, e1600850. https://doi.org/10.1126/sciadv.1600850 (2016)

18. Carmo, E. L., Bueno, A. F. \& Bueno, R. C. O. F. Pesticide selectivity for the insect egg parasitoid Telenomus remus. Biocontrol 55, 455-464. https://doi.org/10.1007/s10526-010-9269-y (2010).

19. Ahmed, N. E., Kanan, H. O., Inanaga, S., Ma, Y. Q. \& Sugimoto, Y. Impact of pesticide seed treatments on aphid control and yield of wheat in the Sudan. Crop Prot. 20, 929-934. https://doi.org/10.1016/S0261-2194(01)00047-3 (2001).

20. Woodford, J. A. T., Gordon, S. C. \& Foster, G. N. Side-band application of systemic granular pesticides for the control of aphids and potato leafroll virus. Crop Prot. 7, 96-105. https://doi.org/10.1016/0261-2194(88)90019-1 (1988).

21. Warnock, D. F. \& Cloyd, R. A. Effect of pesticide mixtures in controlling western flower thrips (Thysanoptera: Thripidae). J. Entomol. Sci. 40, 54-66. https://doi.org/10.18474/0749-8004-40.1.54 (2005).

22. Sawicki, R. M. \& Denholm, I. Management of resistance to pesticides in cotton pests. Trop. Pest Manag. 33, 262-272. https://doi. org $/ 10.1080 / 09670878709371167$ (1987).

23. Costa, L. G., Giordano, G., Guizzetti, M. \& Vitalone, A. Neurotoxicity of pesticides: A brief review. Front. Biosci. Landmark 13, 1240-1249. https://doi.org/10.2741/2758 (2008).

24. Boyles, R. E. et al. Quantitative trait loci mapping of agronomic and yield traits in two grain sorghum biparental families. Crop Sci. 57, 2443-2456 (2017).

25. Kozik, E. \& Sobiczewski, P. Inheritance of sensitivity to fenthion in resistant and susceptible to bacterial speck (Pseudomonas syringae pv. tomato) tomato genotypes. Veg. Crops Res. Bull. 69, 15-19 (2008).

26. Laterrot, H. Susceptibility of the (Pto) plants to Lebaycid insecticide: A tool for breeders. Report of the Tomato Genetics Cooperative (1985).

27. Pedley, K. F. \& Martin, G. B. Molecular basis of Pto-mediated resistance to bacterial speck disease in tomato. Annu. Rev. Phytopathol. 41, 215-243. https://doi.org/10.1146/annurev.phyto.41.121602.143032 (2003).

28. Martin, G. B. et al. A member of the tomato Pto gene family confers sensitivity to fenthion resulting in rapid cell death. Plant Cell 6, 1543-1552. https://doi.org/10.1105/tpc.6.11.1543 (1994).

29. Salmeron, J. M. et al. Tomato Prf is a member of the leucine-rich repeat class of plant disease resistance genes and lies embedded within the Pto kinase gene cluster. Cell 86, 123-133. https://doi.org/10.1016/S0092-8674(00)80083-5 (1996).

30. Mucyn, T. S., Wu, A. J., Balmuth, A. L., Arasteh, J. M. \& Rathjen, J. P. Regulation of tomato Prf by Pto-like protein kinases. Mol. Plant Microbe Interact. 22, 391-401. https://doi.org/10.1094/Mpmi-22-4-0391 (2009).

31. Du, X. R. et al. Plant programmed cell death caused by an autoactive form of Prf is suppressed by co-expression of the Prf LRR domain. Mol. Plant 5, 1058-1067. https://doi.org/10.1093/mp/sss014 (2012).

32. Kimura, K. et al. Surveys of viruliferous alate aphid of Plum pox virus in Prunus mume orchards in Japan. Plant Dis. 100, 40-48. https://doi.org/10.1094/PDIS-05-15-0540-RE (2016).

33. Kim, T. H. et al. Natural variation in small molecule-induced TIR-NB-LRR signaling induces root growth arrest via EDS1- and PAD4-complexed R protein VICTR in Arabidopsis. Plant Cell 24, 5177-5192. https://doi.org/10.1105/tpc.112.107235 (2012).

34. Balota, M., Payne, W. A., Rooney, W. \& Rosenow, D. Gas exchange and transpiration ratio in sorghum. Crop Sci. 48, 2361-2371. https://doi.org/10.2135/cropsci2008.01.0051 (2008).

35. Saballos, A. Genetic Improvement of Bioenergy Crops 211-248 (Springer, 2008).

36. Singh, B. U., Padmaja, P. G. \& Seetharama, N. Biology and management of the sugarcane aphid, Melanaphis sacchari (Zehntner) (Homoptera : Aphididae), in sorghum: A review. Crop Prot. 23, 739-755. https://doi.org/10.1016/j.cropro.2004.01.004 (2004)

37. Hakeem, A. \& Parajulee, M. Integrated management of sugarcane aphid, Melanaphis sacchari (Hemiptera: Aphididae), on sorghum on the Texas high plains. Southwest Entomol. 44, 825-837. https://doi.org/10.3958/059.044.0420 (2019).

38. Colares, F., Michaud, J. P., Bain, C. L. \& Torres, J. B. Recruitment of aphidophagous arthropods to sorghum plants infested with Melanaphis sacchari and Schizaphis graminum (Hemiptera: Aphididae). Biol. Control 90, 16-24. https://doi.org/10.1016/j.bioco ntrol.2015.05.009 (2015). 
39. Kajiya-Kanegae, H. et al. RAD-seq-based high-density linkage map construction and QTL mapping of biomass-related traits in sorghum using the Japanese Landrace Takakibi NOG. Plant Cell Physiol. 61, 1262-1272. https://doi.org/10.1093/pcp/pcaa056 (2020).

40. Takanashi, H. et al. Genetic dissection of QTLs associated with spikelet-related traits and grain size in sorghum. Sci. Rep. UK 11, 9398. https://doi.org/10.1038/s41598-021-88917-x (2021).

41. Ohnishi, N., Wacera, W. F. \& Sakamoto, W. Photosynthetic responses to high temperature and strong light suggest potential postflowering drought tolerance of sorghum Japanese Landrace Takakibi. Plant Cell Physiol. 60, 2086-2099. https://doi.org/10.1093/ pcp/pcz107 (2019).

42. Tao, Y., Jordan, D., Henzell, R. \& McIntyre, C. Construction of a genetic map in a sorghum recombinant inbred line using probes from different sources and its comparison with other sorghum maps. Aust. J. Agric. Res. 49, 729-736 (1998).

43. Tao, Y. et al. Identification of genomic regions associated with stay green in sorghum by testing RILs in multiple environments. Theor. Appl. Genet. 100, 1225-1232 (2000).

44. Mace, E. S. \& Jordan, D. R. Location of major effect genes in sorghum (Sorghum bicolor (L.) Moench). Theor. Appl. Genet. 121, 1339-1356. https://doi.org/10.1007/s00122-010-1392-8 (2010).

45. Xu, W. et al. Molecular mapping of QTLs conferring stay-green in grain sorghum (Sorghum bicolor L. Moench). Genome 43, 461-469 (2000).

46. Kawahigashi, H., Mizuno, H., Ando, T., Yonemaru, J. I. \& Kasuga, S. Molecular mapping and identification of candidate gene conferring organophosphate-Sensitive reaction in sorghum (Sorghum bicolor). Plant Breed. 139, 600-607 (2020).

47. Salanoubat, M. et al. Sequence and analysis of chromosome 3 of the plant Arabidopsis thaliana. Nature 408, 820-822. https://doi. org/10.1038/35048706 (2000).

48. Du, D. et al. The CC-NB-LRR OsRLR1 mediates rice disease resistance through interaction with OsWRKY19. Plant Biotechnol. J. https://doi.org/10.1111/pbi.13530 (2020).

49. Shang, J. et al. Identification of a new rice blast resistance gene, Pid3, by genomewide comparison of paired nucleotide-binding site-leucine-rich repeat genes and their pseudogene alleles between the two sequenced rice genomes. Genetics 182, 1303-1311. https://doi.org/10.1534/genetics.109.102871 (2009).

50. Mugnozza, G. S., Porceddu, E. \& Pagnotta, M. In Genetics and Breeding for Crop Quality and Resistance: Proceedings of the XV Eucarpia Congress, Viterbo, Italy, September 20-25, 1998, 103 (Springer).

51. Salmeron, J. M., Barker, S. J., Carland, F. M., Mehta, A. Y. \& Staskawicz, B. J. Tomato mutants altered in bacterial disease resistance provide evidence for a new locus controlling pathogen recognition. Plant Cell 6, 511-520. https://doi.org/10.1105/tpc.6.4.511 (1994).

52. Kawahigashi, H. et al. Positional cloning of ds1, the target leaf spot resistance gene against Bipolaris sorghicola in sorghum. Theor. Appl. Genet. 123, 131-142. https://doi.org/10.1007/s00122-011-1572-1 (2011).

53. Kimball, J. et al. Identification of QTL for Target Leaf Spot resistance in Sorghum bicolor and investigation of relationships between disease resistance and variation in the MAMP response. Sci. Rep. UK 9, 18285. https://doi.org/10.1038/s41598-019-54802-x (2019).

54. Mackey, D., Holt, B. F. 3rd., Wiig, A. \& Dangl, J. L. RIN4 interacts with Pseudomonas syringae type III effector molecules and is required for RPM1-mediated resistance in Arabidopsis. Cell 108, 743-754. https://doi.org/10.1016/s0092-8674(02)00661-x (2002).

55. Adachi, H., Derevnina, L. \& Kamoun, S. NLR singletons, pairs, and networks: evolution, assembly, and regulation of the intracellular immunoreceptor circuitry of plants. Curr. Opin. Plant Biol. 50, 121-131. https://doi.org/10.1016/j.pbi.2019.04.007 (2019).

56. Broman, K. W., Wu, H., Sen, S. \& Churchill, G. A. R/qtl: QTL mapping in experimental crosses. Bioinformatics 19, 889-890. https:// doi.org/10.1093/bioinformatics/btg112 (2003).

57. Kosambi, D. D. DD Kosambi 125-130 (Springer, 2016).

58. Haley, C. S. \& Knott, S. A. A simple regression method for mapping quantitative trait loci in line crosses using flanking markers. Heredity 69, 315-324. https://doi.org/10.1038/hdy.1992.131 (1992).

59. Saitou, N. \& Nei, M. The neighbor-joining method: A new method for reconstructing phylogenetic trees. Mol. Biol. Evol. 4, 406-425. https://doi.org/10.1093/oxfordjournals.molbev.a040454 (1987).

60. Zuckerkandl, E. \& Pauling, L. Evolving Genes and Proteins 97-166 (Elsevier, 1965).

61. Kumar, S., Stecher, G., Li, M., Knyaz, C. \& Tamura, K. MEGA X: Molecular evolutionary genetics analysis across computing platforms. Mol. Biol. Evol. 35, 1547-1549. https://doi.org/10.1093/molbev/msy096 (2018).

62. Kawano, Y. et al. Activation of a Rac GTPase by the NLR family disease resistance protein Pit plays a critical role in rice innate immunity. Cell Host Microbe 7, 362-375. https://doi.org/10.1016/j.chom.2010.04.010 (2010).

\section{Acknowledgements}

We thank Makoto Ishii, Masaru Fujimoto, Rie Hijiya, Norikazu Ohnishi, and Adhiambo Everlyne Omollo for their technical assistance and discussions in performing this study. This work was partly supported by KAKENHI grants (17H03699, 18K19343, and 21H02508 to W.S.) from the Japan Society for the Promotion of Science (JSPS). We also thank the Oohara Foundation for financial support of our research group.

\section{Author contributions}

W.S. designed the experiment in consultation with H.K.-K., H.T., H.I. N.T.; Z.J., F.W.W., T.T., F.F., Y.K., H.T., H.K-K., and W.S. performed the experiments; Z.J., F.W.W., H.K.-K., and W.S. analyzed data; Z.J. and W.S. wrote the manuscript on behalf of all authors.

\section{Competing interests}

The authors declare no competing interests.

\section{Additional information}

Supplementary Information The online version contains supplementary material available at https://doi.org/ 10.1038/s41598-021-98908-7.

Correspondence and requests for materials should be addressed to W.S.

Reprints and permissions information is available at www.nature.com/reprints.

Publisher's note Springer Nature remains neutral with regard to jurisdictional claims in published maps and institutional affiliations. 
(c) (i) Open Access This article is licensed under a Creative Commons Attribution 4.0 International cc) License, which permits use, sharing, adaptation, distribution and reproduction in any medium or format, as long as you give appropriate credit to the original author(s) and the source, provide a link to the Creative Commons licence, and indicate if changes were made. The images or other third party material in this article are included in the article's Creative Commons licence, unless indicated otherwise in a credit line to the material. If material is not included in the article's Creative Commons licence and your intended use is not permitted by statutory regulation or exceeds the permitted use, you will need to obtain permission directly from the copyright holder. To view a copy of this licence, visit http://creativecommons.org/licenses/by/4.0/.

(C) The Author(s) 2021 